\title{
Transcranial Electrical Stimulation as a Tool to Enhance Attention
}

\author{
L. C. Reteig ${ }^{1,2,3}$ - L. J. Talsma ${ }^{1,2}$ • M. R. van Schouwenburg ${ }^{1,2}$ • H. A. Slagter ${ }^{1,2}$
}

Received: 24 September 2016 / Accepted: 9 February 2017 / Published online: 10 March 2017

(C) The Author(s) 2017. This article is published with open access at Springerlink.com

\begin{abstract}
Attention is a fundamental cognitive process-without it, we would be helplessly adrift in an overload of sensory input. There is considerable interest in techniques that can be used to enhance attention, including transcranial electrical stimulation (tES). We present an overview of 52 studies that have paired attention tasks with tES, mostly in the form of transcranial direct current stimulation (tDCS). In particular, we discuss four aspects of attention that have been most extensively targeted to date: visual search, spatial orienting (e.g., Posner cueing tasks), spatial bias (e.g., line bisection tasks), and sustained attention. Some promising results have been reported in each of these domains. However, drawing general conclusions about the efficacy of tES is at present hampered by a large diversity in study design and inconsistent findings. We highlight some pitfalls and opportunities and suggest how these may be addressed in future research aiming to use tES as a tool to enhance or test theoretical hypotheses about attention.
\end{abstract}

Keywords transcranial direct current stimulation (tDCS) . transcranial alternating current stimulation (tACS) . Cognition $\cdot$ Cognitive enhancement $\cdot$ Spatial attention . Neglect $\cdot$ Sustained attention

L. C. Reteig

1.c.reteig@uva.nl

1 Department of Psychology, University of Amsterdam, Nieuwe Achtergracht 129-B, 1018 WS Amsterdam, The Netherlands

2 Amsterdam Brain and Cognition, University of Amsterdam, Nieuwe Achtergracht 129-B, 1018 WS Amsterdam, The Netherlands

3 PO Box 15915, 1001 NK Amsterdam, The Netherlands

\section{Introduction}

Attention - the ability to prioritize processing of goal-relevant information - underpins many of our daily behaviors. Attentional disturbances lie at the core of many psychiatric and neurological disorders, such as ADHD and hemineglect. It is therefore not surprising that attention has been a primary focus of cognitive enhancement techniques, ranging from pharmacological stimulants (Koelega 1993) to video games (Green and Bavelier 2012) and meditation training (Lutz et al. 2008).

The recent rediscovery of transcranial electrical stimulation (tES) has added another technique to the arsenal. tES comprises a family of methods in which a weak current is run between electrodes placed on the skin, partly passing through the skull and changing the excitability of the underlying brain tissue.

In transcranial direct current stimulation (tDCS) - the most widely used form of tES - the current flows in one direction: from the (positive) anode to the (negative) cathode. tDCS has been used to enhance a number of cognitive abilities (Cohen Kadosh 2014; Coffman et al. 2014; Mancuso et al. 2016; but see Horvath et al. 2015a).

While the neurophysiological mechanisms of tDCS remain to be fully uncovered (for reviews, see Stagg and Nitsche 2011, and Medeiros et al. 2012), its effects during stimulation are generally attributed to changes in the resting membrane potential of neurons. Anodal stimulation typically depolarizes the resting membrane potential, bringing neurons closer to their firing threshold, while cathodal stimulation generally decreases neuronal excitability (Nitsche et al. 2008). This finding is backed up by in vitro (Terzuolo and Bullock 1956), animal (Bindman et al. 1964), and human motor-evoked potential studies (Nitsche and Paulus 2000, 2001). 
However, the anode-excitation and cathode-inhibition dichotomy is dependent on many factors and does not necessarily extrapolate to all cases (Bestmann et al. 2015; Jacobson et al. 2012; Parkin et al. 2015). Even very low-level factors can influence the precise neurophysiological effects of tDCS. For example, individual differences in the cortical folding pattern lead to differences in local current density (Opitz et al. 2015) and the orientation of neurons with respect to the electric field (Radman et al. 2009).

The effects of tDCS are not confined to the stimulation period, but can outlast it for minutes to hours, or even months after multiple stimulation sessions (Snowball et al. 2013), probably by promoting neural plasticity. These aftereffects appear to involve many different stages and effectors, but glutamate and GABA concentrations might play a central role (Stagg and Nitsche 2011). Anodal tDCS has been associated with increases in glutamate (Clark et al. 2011; Hone-Blanchet et al. 2016), while cathodal tDCS has been linked to decreases in both glutamate and GABA levels (Stagg et al. 2009). Because the immediate and aftereffects of tDCS involve such different neural mechanisms, studies that apply online stimulation (during a cognitive task) are not necessarily comparable to offline studies (where tDCS is applied before the task).

Instead of applying direct current, the polarity of the electrodes (anode or cathode) can also be switched at a certain frequency. This method, known as transcranial alternating current stimulation (tACS), effectively injects an oscillatory current into the brain that might entrain endogenous neural oscillations (Herrmann et al. 2013). As oscillations play an important role in cognitive processes (Buzsáki and Draguhn 2004; Siegel and Donner 2010), including attention (Buschman and Miller 2007; Womelsdorf and Fries 2007), tACS may offer a more specific means to affect attention. Finally, transcranial random noise stimulation (tRNS) applies a whole spectrum of frequencies at once (e.g., 0.1-640 Hz). tRNS is believed to enhance excitability and promote neuroplasticity under both electrodes (Antal and Herrmann 2016; Terney et al. 2008).

We aim to provide a comprehensive overview of studies conducted to date that used tES to modulate attention. After a systematic search and screening of the results, we included 52 studies. Details on our search query, inclusion criteria, and excluded studies are available on the Open Science Framework https://osf.io/kqvap/. A summary of the methods and results of the included studies is presented in the next sections and in Tables 1, 2, 3, and 4, clustered by the most frequently used types of tasks: visual search, spatial orienting, spatial bias, and sustained attention. Each of these tasks taps into distinct attentional processes that are relevant in different real-life situations. Our selection also included six studies that did not fit into one of these four categories (Table 5).

As our overview reveals, there is tremendous variability in design and stimulation parameters between studies, and in study outcome, hampering integration and interpretation of different results. The scope of this review did not allow an extensive discussion of each study or differences between them. To remain as comprehensive as possible, we chose to catalogue the details of each study in the tables and to summarize the main conclusions in the body text. We hope that this way our review will prove useful to a broad audiencethose looking for the gist as well as those interested in fine details, perhaps while preparing to set up a new experiment of their own. To this end, we also provide more general recommendations for future research in the "Discussion" section.

\section{Visual Search}

Whether you are looking for a particular pair of socks or crossing a busy intersection, the process of scanning the visual field is ubiquitous in daily life and therefore an interesting target for cognitive enhancement. We included 13 studies that examined the effects of tES on visual search (Table 1).

In visual search tasks, participants look for a target item among an array of distractors (Wolfe 1998). This involves keeping a template of the target online and shifting spatial attention across the visual field, while constantly filtering out distracting information. Faster reaction times on these tasks indicate more efficient visual search. Visual search performance is supported by an extensive network of brain areas, centered on the right posterior parietal cortex and frontal eye field (Reynolds and Chelazzi 2004).

Ball et al. (2013) investigated the effect of anodal and cathodal tDCS on both of these areas. They observed no effects of anodal tDCS, but cathodal stimulation to the right posterior parietal cortex increased reaction times, a finding later replicated by the same group (Ellison et al. 2014). Interestingly, an earlier study did find that anodal stimulation over the parietal cortex decreased search time (Bolognini et al. 2010a). As the studies differ in many design choices, it is difficult to tell what may account for these inconsistent results.

More recent studies suggest that stimulation effects on visual search depend on target location and distractor type. For example, whereas anodal tDCS may improve performance for targets contralateral to the stimulated hemisphere, it may worsen performance for ipsilateral targets (Reinhart and Woodman 2015). In another study, the effect of tDCS was contingent on the presence of a salient distractor: tDCS increased distractor resistance but did not improve visual search in general (Cosman et al. 2015).

Two other studies also observed effects specific to distractor processing. One study found that cathodal tDCS to the right parietal cortex increased the effect of task-irrelevant flanker stimuli on performance, specifically for difficult searches (Weiss and Lavidor 2012). Another study was unable to replicate this result, but found that in fact anodal tDCS decreased the flanker effect for easy searches (Kajimura and 


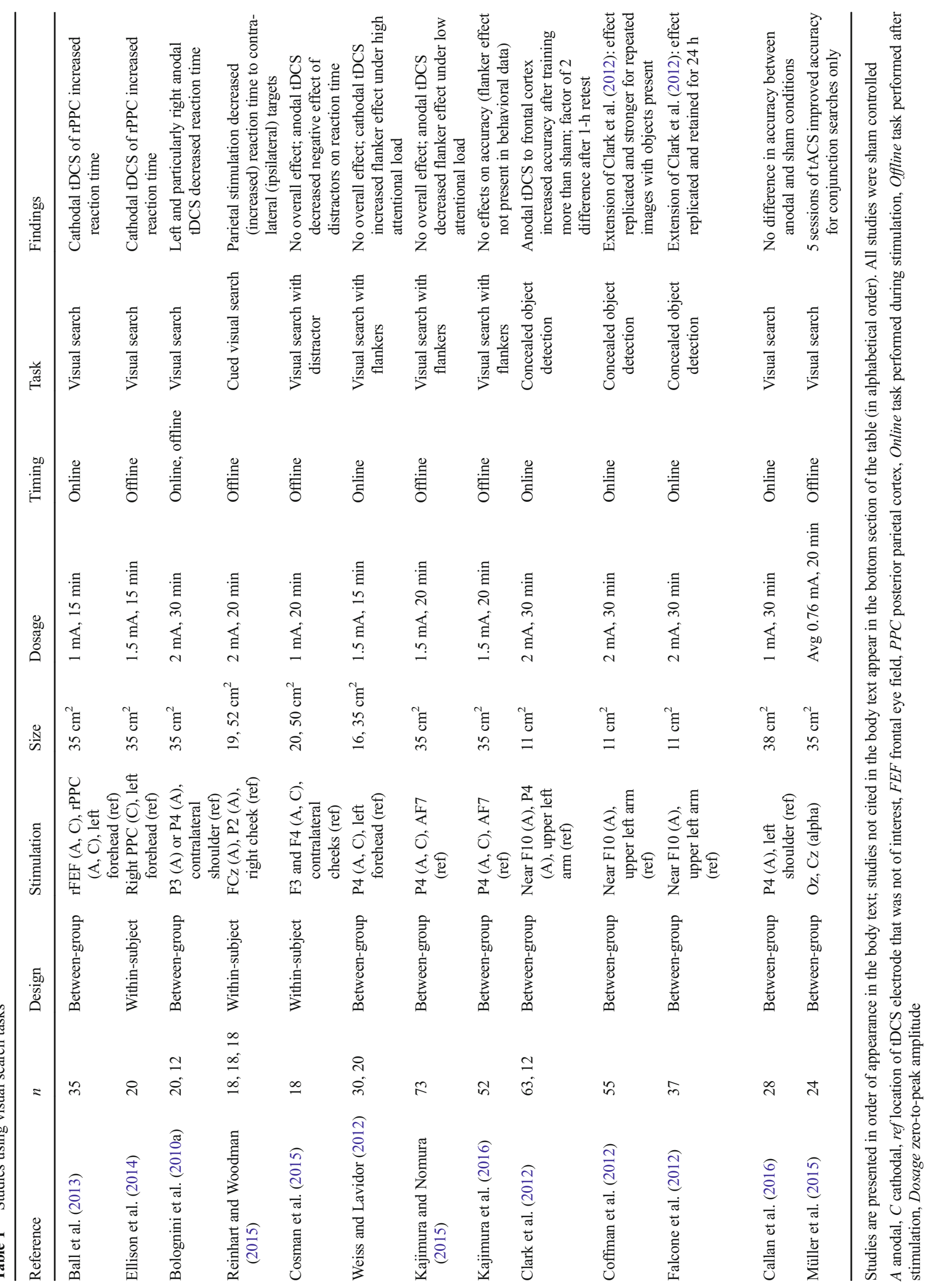


Nomura 2015). These inconsistencies could be due to a difference in stimulation timing-Weiss and Lavidor (2012) applied online tDCS; Kajimura and Nomura (2015) stimulated offline - but otherwise these two studies used highly similar tasks and stimulation protocols.

The largest and most consistent effects of tDCS on visual search processes hail from a series of studies by Clark and colleagues. They found that learning to detect concealed threatening objects embedded in naturalistic scenes was greatly enhanced by anodal tDCS over the right inferior frontal cortex - up to a factor of 2 compared to sham stimulation (Clark et al. 2012). Subsequent studies replicated this effect (Coffman et al. 2012) and showed it is retained for at least $24 \mathrm{~h}$ (Falcone et al. 2012).

In conclusion, anodal tDCS over the right parietal cortex may speed up visual search, while cathodal stimulation may slow it down. However, results are inconsistent, may differ per hemifield, and appear restricted to particular aspects of visual search (e.g., salient distractors). Anodal stimulation over the right inferior frontal cortex has consistently shown to speed up object detection. Perhaps this success can be attributed to the challenging nature of the task: tES effects might be greatest for difficult tasks with plenty of room for improvement (e.g., Jones and Berryhill 2012). Because this task is more complex than the typical visual search paradigm, it could also be that tDCS affected other processes such as threat detection or scene perception, and not those underlying visual search per se.

\section{Spatial Orienting}

A second aspect of attention implicit in visual search is visuospatial orienting: the ability to allocate spatial attention to relevant parts of the visual field. Visuospatial orienting can be driven by a target stimulus itself (stimulus-driven orienting) or by spatial cues that prompt automatic (exogenous) or voluntary (endogenous) orienting towards or away from the upcoming target. In total, we identified 12 studies that evaluated tES effects on spatial orienting (Table 2).

\section{Stimulus-Driven Orienting}

The effects of tES on stimulus-driven orienting can be studied by stimulating the parietal cortex ipsi- or contralateral to a target stimulus. The assumption is that increasing the excitability of the contralateral hemisphere with anodal tDCS might shift attention towards the relevant visual field - thus improving accuracy or reaction times for target detectionwhile ipsilateral stimulation should have the opposite effect.

To test this, Bolognini et al. (2010b) briefly presented target stimuli either to the left or right part of the visual field concurrent with anodal tDCS to the right parietal cortex. Indeed, they report a decrease in reaction time for targets presented in the left (contralateral), but not the right hemifield (ipsilateral). Likewise, contralateral target detection was also found to improve with anodal stimulation in another study (Sparing et al. 2009). However, Filmer et al. (2015) found diminished performance for both contralateral and bilateral stimuli following anodal tDCS. This discrepancy in findings is unexpected as the design of the latter two studies is quite similar, although both did employ fairly low-dosage stimulation.

Cathodal stimulation over the parietal cortex increased performance for ipsilateral targets (Sparing et al. 2009), but decreased performance for contralateral targets and also bilateral stimuli (Filmer et al. 2015; Sparing et al. 2009). Possibly, the representation of the ipsilateral stimulus is enhanced by cathodal stimulation, captures attention, and biases awareness to just that one stimulus.

Interestingly, tES might not affect orienting similarly in everyone. Learmonth et al. (2015) found no group effects of stimulation, but splitting their sample according to baseline performance revealed a weak impairment with anodal tDCS: both accuracy and reaction time worsened, but only in participants with already low baseline task performance.

To summarize, although some studies report unilateral modulations in the expected direction, the overall findings of studies were not consistent. Stimulation did not necessarily improve attention, but sometimes also led to decrements in one or both hemifields. Future studies could examine whether enhancing the excitability of both hemispheres at once with tRNS or two anodes is more effective and might circumvent these impairments.

\section{Endogenous and Exogenous Orienting}

Spatial cueing tasks, such as the classical Posner paradigm, can chart how spatial attention prior to stimulus presentation facilitates performance (Chica et al. 2014; Posner 1980). In the endogenous (or top-down) variant, attention is willfully directed to the left or right hemifield following a central cue. In exogenous (or bottom-up) orienting, attention is automatically drawn to a location in the left or right hemifield with a peripheral cue. On trials with valid cues, a target is presented at the cued location; on trials with invalid cues, the target is presented at the uncued location. While endogenous orienting is associated with activity in a more dorsal frontoparietal network, a mostly right-lateralized network of ventral frontal and parietal areas underpins exogenous orienting (Corbetta and Shulman 2002).

tDCS studies with Posner tasks have yielded markedly different results. Anodal tDCS to the right parietal cortex improved performance, but surprisingly for both valid and invalid endogenous cues (Bolognini et al. 2010a). A more recent study in which both hemispheres received opposite polarity stimulation did not identify any significant effect on 

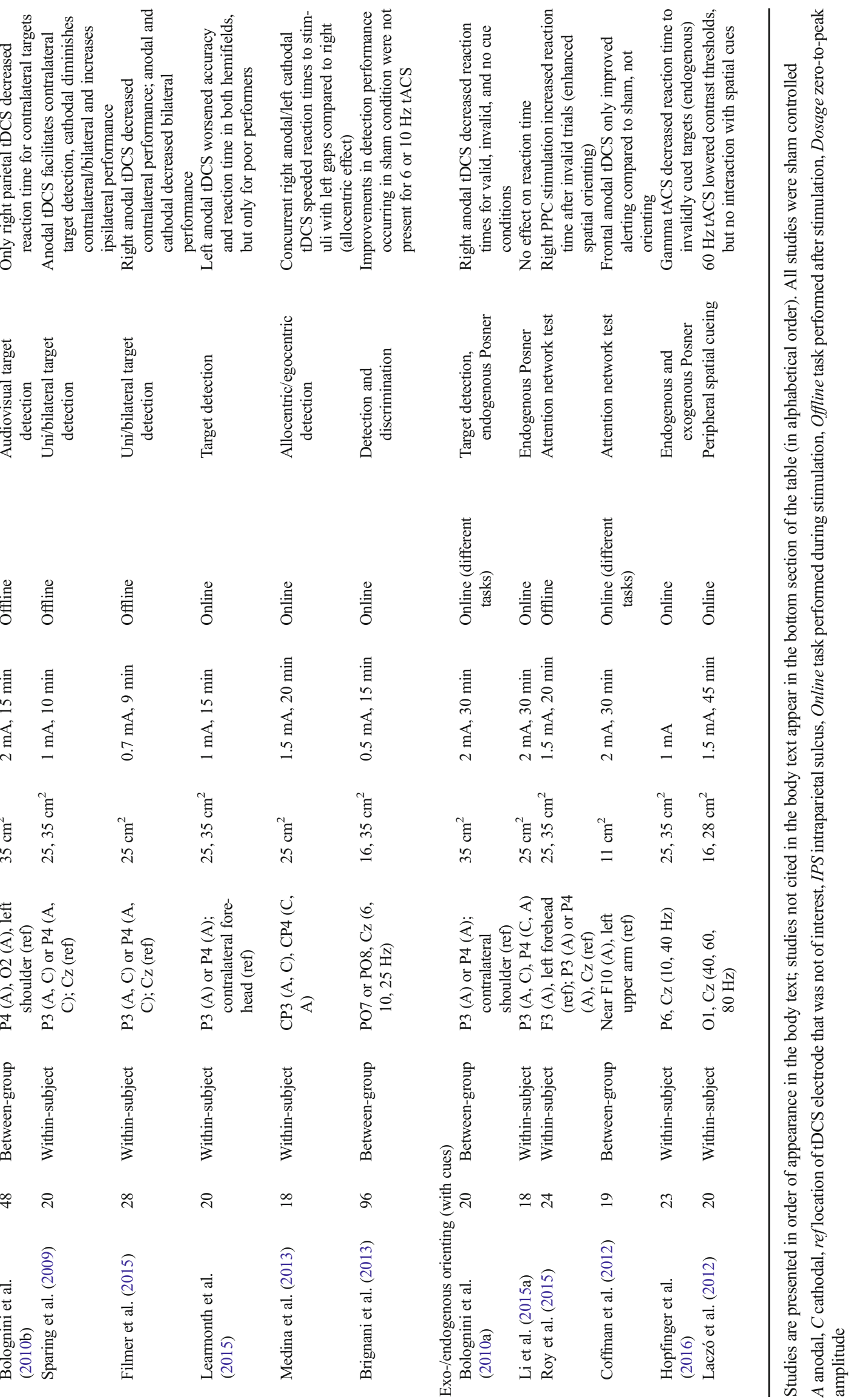

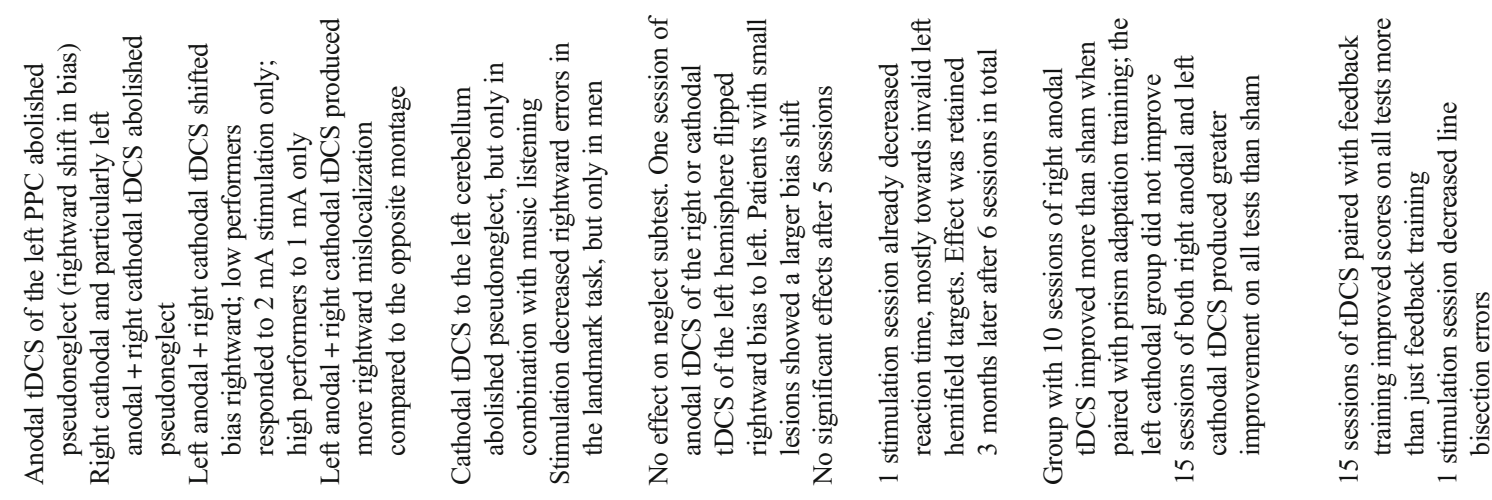

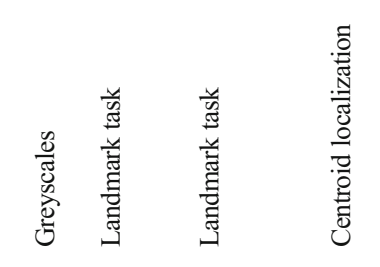
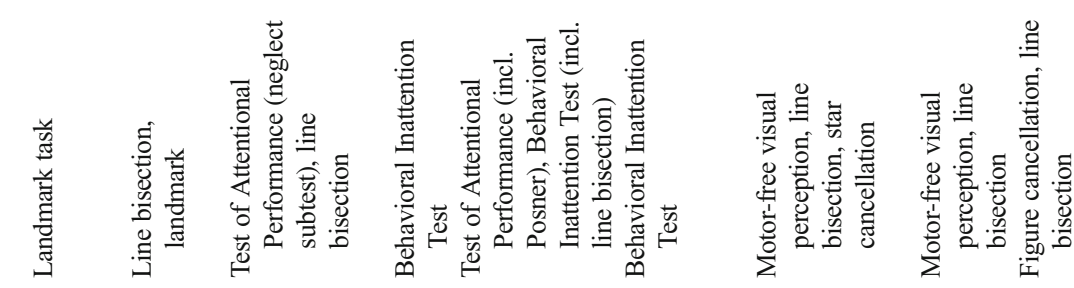
妾

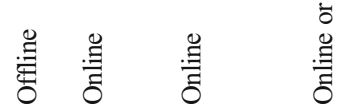

亭 量 量

甾兽

章 咅

旁 营

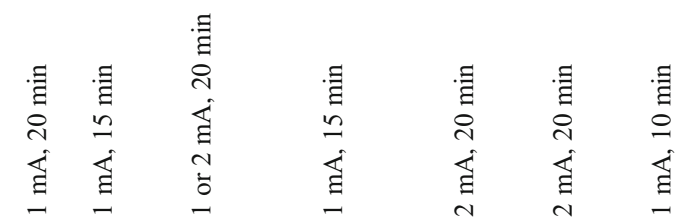

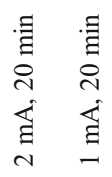

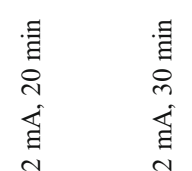

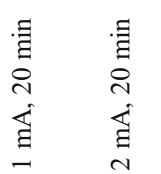

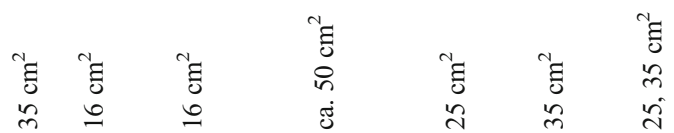

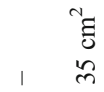

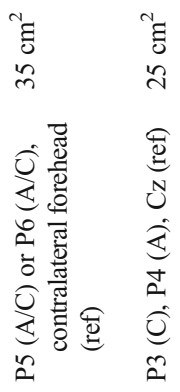

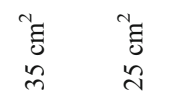

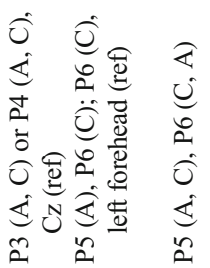

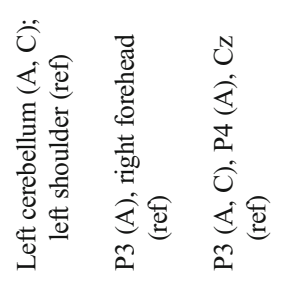

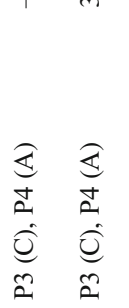

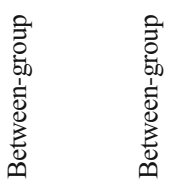

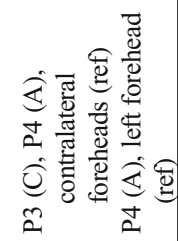

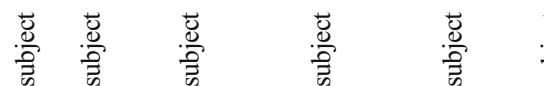

苛

요

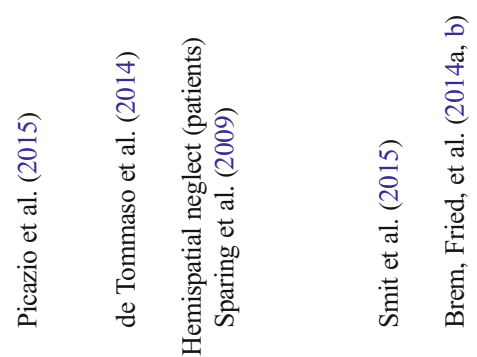

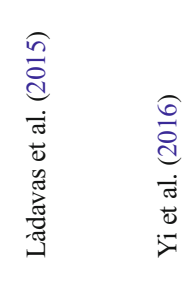

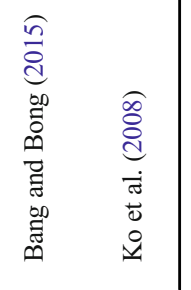


performance ( $\mathrm{Li}$ et al. 2015a). Using a more comprehensive version of Posner's paradigm that also measures alerting and executive attention - the attention network test (Fan et al. 2002) - Roy et al. (2015) found that anodal tDCS to the right parietal cortex decreased performance after invalid exogenous cues. Stimulation over the right inferior frontal cortex did not seem to affect orienting in the attention network test (Coffman et al. 2012), although here, participants did not perform the test until $1.5 \mathrm{~h}$ after stimulation offset.

Two studies so far have paired tACS with a spatial cueing task. Laczó et al. (2012) found that while $60 \mathrm{~Hz}$ (gammaband) tACS over the visual cortex improved contrast perception, this effect was not modulated by exogenous spatial cues. A recent study found no effect on exogenous cues, but gamma tACS over the right parietal cortex did decrease reaction times following invalid, endogenous cues (Hopfinger et al. 2016).

In conclusion, whether preceded by a spatial cue or not, anodal tDCS to the right parietal cortex did not consistently enhance processing of left visual field stimuli. Several studies even found performance decreases (Filmer et al. 2015; Learmonth et al. 2015). Not a single study reported a benefit to the endogenous control of attention. Future studies should aim to replicate these (null) findings and explore different nodes of the topdown (e.g., the frontal eye fields) or bottom-up (e.g., the temporoparietal junction) attention networks (Corbetta and Shulman 2002).

\section{Spatial Bias}

A closely related line of studies has used tES to modify spatial biases in attention, both in the healthy and diseased brains. These studies capitalize on the finding that attention is not symmetrically distributed over the visual field. Most people exhibit pseudoneglect: they overemphasize features in the left versus the right hemifield (Jewell and McCourt 2000). This bias likely occurs because the right hemisphere is slightly more active than the left at rest, thus shifting attention towards the left visual hemifield. Spatial biases can be quantified with line bisection tests (Bowers and Heilman 1980), in which people typically bisect the line slightly towards the left. In contrast to the naturally occurring pseudoneglect, hemispatial neglect is a much more extreme bias that can occur in stroke patients. We identified 15 studies examining the effect of tDCS on both of these biases, which have produced relatively consistent results (Table 3).

\section{Pseudoneglect}

Loftus and Nicholls (2012) demonstrated that pseudoneglect can be reversed with anodal tDCS over the left parietal cortex. 


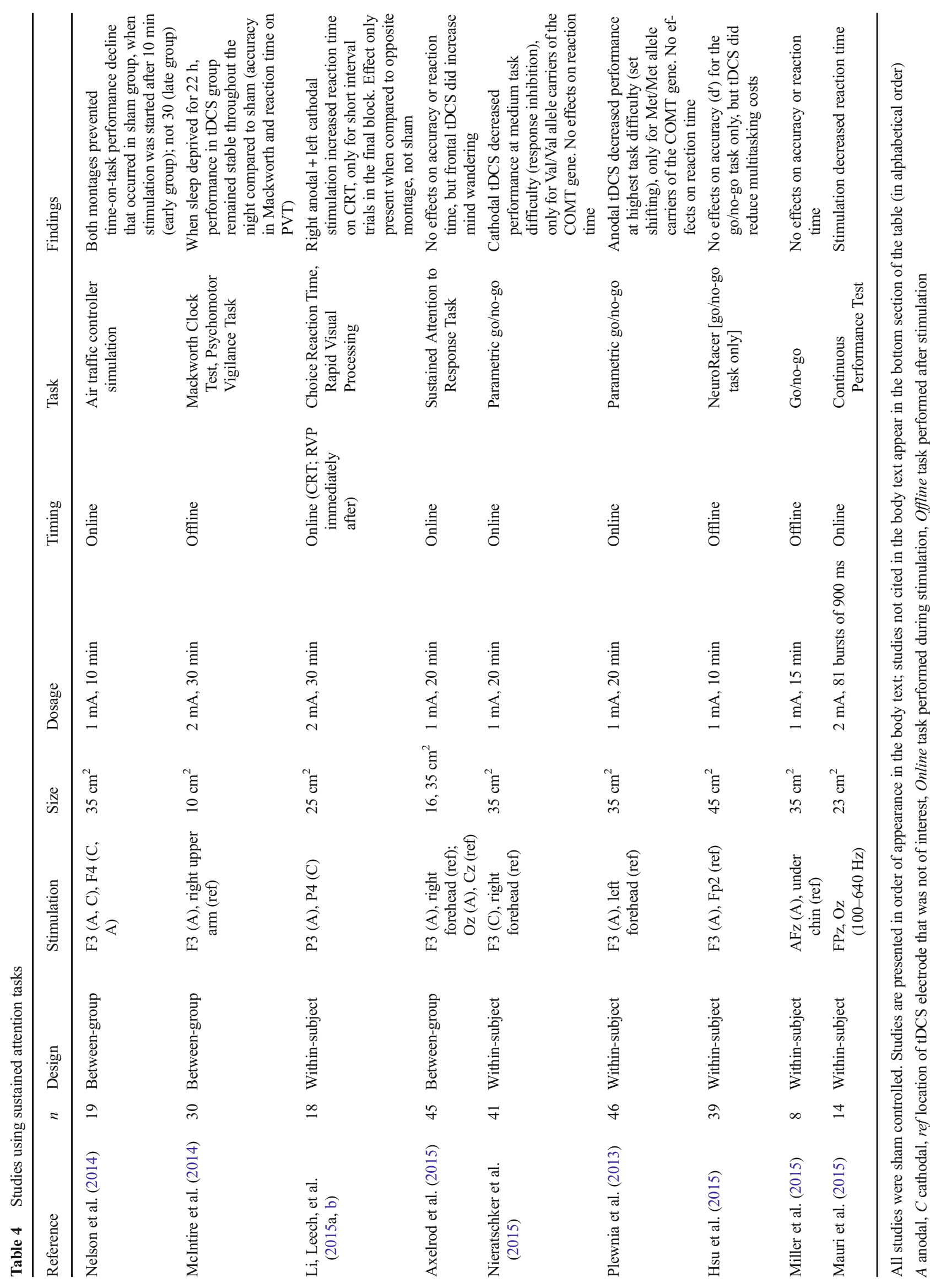




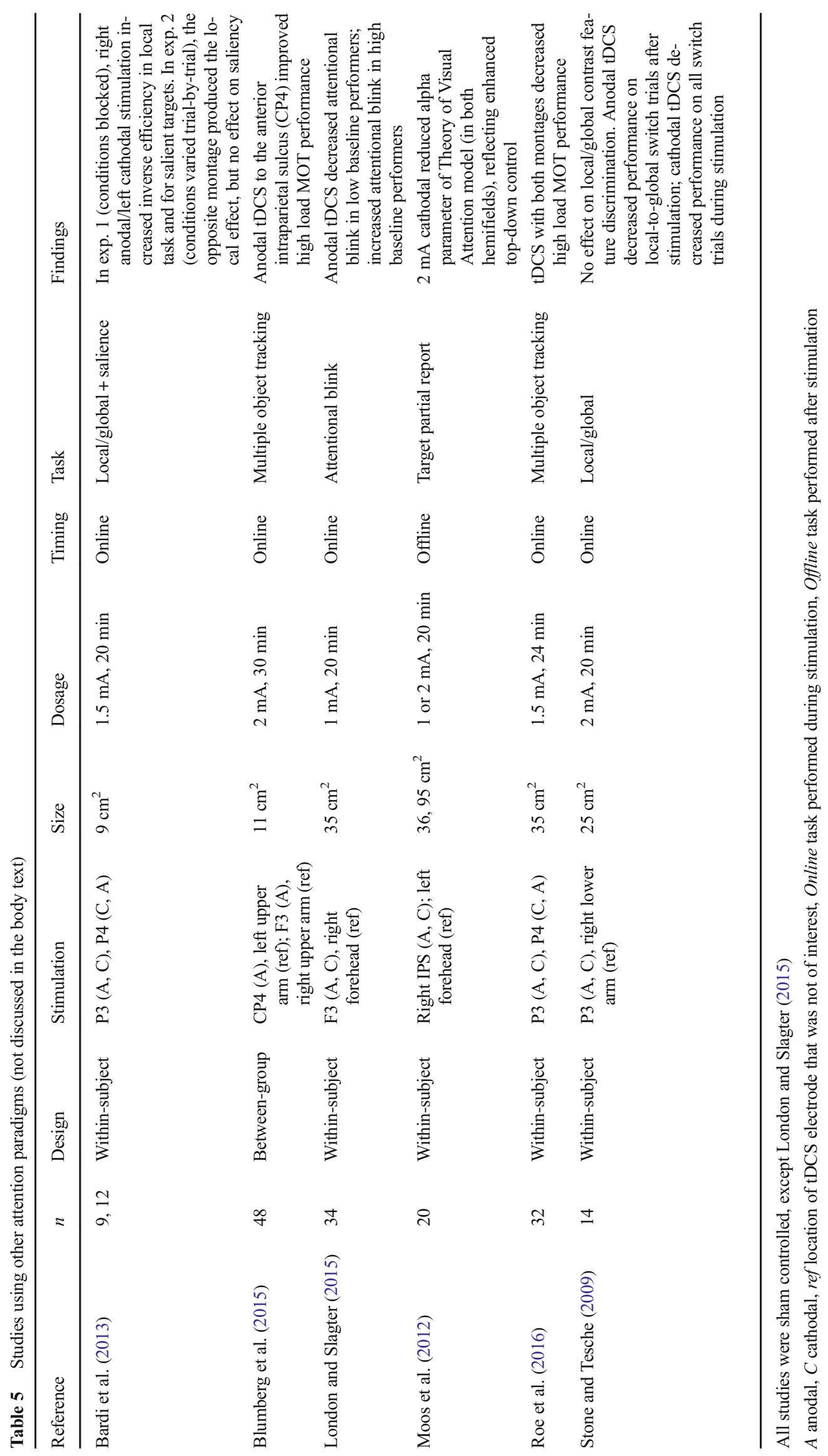


Presumably, tDCS increased the activity of the left parietal cortex beyond that of the right, causing a rightward shift in spatial bias. Similarly, Giglia et al. (2011) report a rightward shift for right cathodal tDCS (although note that Loftus and Nicholls (2012) did not find this). They furthermore observed that a "dual" montage with one electrode on each posterior parietal cortex (anode left; cathode right) was even more effective.

This dual montage effect was replicated with a larger sample, although the overall effect size was fairly small (Benwell et al. 2015). An exploratory analysis revealed that the effect was strongly modulated by individual differences: those who performed well at baseline responded only to weaker current intensity ( $1 \mathrm{~mA})$; those who did poorly at baseline responded only to $2 \mathrm{~mA}$ tDCS. This pattern makes sense: those with a large bias to begin with likely had a more active right hemisphere at baseline, so higher intensity stimulation would be needed to tip the scales.

In conclusion, anodal tDCS to the left and cathodal tDCS to the right parietal cortex appear to shift spatial bias rightwards, though the effect could be subject to individual differences, which were also present in the other three studies (Table 3).

\section{Hemispatial Neglect}

Hemispatial neglect occurs most frequently following lesions of the right ventral parietal cortex (Vallar and Perani 1986). Neglect patients have difficulty to voluntarily orient attention to the visual hemifield contralateral to the lesion (Heilman et al. 2012) and thus exhibit a spatial bias to the ipsilateral hemifield (most often right). This bias is related to hypoactivity of the ipsilateral (right) parietal cortex and hyperactivity of the contralateral (left) parietal cortex (Corbetta et al. 2005). tES might be uniquely suitable to restore this interhemispheric imbalance by either increasing excitability of the lesioned hemisphere and/or inhibiting the nonlesioned hemisphere.

Nine studies have investigated this prospect to date, with promising results (Table 3). For example, Sparing et al. (2009) administered anodal tDCS to the right parietal cortex or cathodal tDCS to the left parietal cortex in neglect patients with right hemisphere lesions. Both protocols abolished the rightward bias in line bisection and produced a small leftward bias. Seven of the other studies also report some improvement after parietal tDCS, many on line bisection tasks.

Notably, one study did not find any effect of tDCS (Smit et al. 2015). Possibly this is due to their low sample size $(n=$ 5) - a problem that plagues all these studies - or because their patients were in the later, chronic stage of stroke, when recovery is more difficult. Still, the null result by Smit et al. (2015) carries some weight, as their study was relatively well-designed: it included multiple patients, was sham-controlled, and had multiple (five) stimulation sessions - only two other studies fit all these criteria (Làdavas et al. 2015; Yi et al. 2016).

Although promising, these initial results should therefore be interpreted with some caution. Moreover, follow-up tests are crucial to assess whether the stimulation effects have any long-term clinical value. Only Brem et al. (2014b) had a 3month follow-up, but treated just a single patient. Still, the current findings warrant a large-scale clinical trial to determine the efficacy of tDCS for treatment of hemispatial neglect.

\section{Sustained Attention}

The final aspect of attention that multiple tES studies have targeted is sustained attention. In sustained attention paradigms, participants continuously monitor a stimulus stream for targets. Typically, after prolonged time-on-task, performance declines rapidly - the so-called vigilance decrement (Mackworth 1948; Parasuraman 1979). Finding ways to counter the vigilance decrement is especially pertinent given that these tasks mirror many real-life situations, such as air traffic control, surveillance, and quality control.

We found nine studies that examined effects of tES on sustained attention (Table 4). Nelson et al. (2014) report that the vigilance decrement could be prevented by applying bilateral tDCS to the dorsolateral prefrontal cortex early into a vigilance task. Prefrontal tDCS may also counter the effects of sleep deprivation on vigilance, to the same or greater extent than caffeine (McIntire et al. 2014). When bilateral stimulation was applied to parietal cortex, Li et al. (2015a) found that tDCS only affected performance on the final block of a reaction time task, but this effect reflected slower instead of faster reaction times.

Other studies that were not geared towards time-on-task effects have reported mixed results. For example, prefrontal tDCS did not affect performance on a sustained attention to response task, but did increase mind wandering (Axelrod et al. 2015). Two other studies employing a go/no-go task found that tDCS negatively affected performance, but only when increased demand was placed on inhibitory control (Nieratschker et al. 2015) and set shifting abilities (Plewnia et al. 2013). However, these effects were restricted to carriers of particular subtypes of the COMT gene, involved in regulation of dopamine levels. Still, this lends some support to the idea that tDCS effects are most apparent at higher levels of task difficulty. Similarly, Hsu et al. (2015) only found effects in a multitasking context, not when participants were performing a single task. These findings collectively suggest that prefrontal tDCS primarily affects higher-order processes involved in sustained attention, and not simple target detection per se. 
In conclusion, while most studies found no or restricted effects on sustained attention, two studies report that prefrontal tDCS specifically offsets the vigilance decrement, suggesting that its effects may only become apparent after prolonged task performance. If replicated, it may be interesting for future studies to investigate the cognitive mechanisms at work: does tDCS allow fatigued individuals to tap into additional attentional resources, or do they simply become more motivated or less prone to mind wandering?

\section{Discussion}

Each of the four aspects of attention reviewed here harbors both promising results and many inconsistencies, such that compelling conclusions cannot be drawn at this point. Anodal stimulation to the right parietal and frontal cortex might speed up learning and reaction time in visual search, but robust results were only reported by one group for one particular visual search task (Clark et al. 2012; Coffman et al. 2012; Falcone et al. 2012). For spatial attention, parietal tDCS may enhance visuospatial processing, but many studies also reported null results or even performance decrements. Prefrontal tDCS may improve sustained attention by countering the performance decrements normally observed after prolonged time-on-task (Nelson et al. 2014; McIntire et al. 2014), but this effect remains to be replicated independently, and tDCS did not produce consistent effects in other sustained attention tasks. The most exciting and consistent results may be those showing that tDCS can shift spatial biases and thereby ameliorate symptoms in hemispatial neglect patients. However, even this field is not without its contradictory results, and it remains to be seen whether this effect stands up to well-controlled and larger clinical trials.

We proceed to discuss several factors that may contribute to the diversity in observed results. We also offer recommendations for future studies that may help resolve these inconsistencies and shed more definitive light on the ability to use tES to enhance attention. As effects of tES on other domains also appear less robust than initially thought (e.g., Horvath et al. 2015b; López-Alonso et al. 2014, 2015; Mancuso et al. 2016), our recommendations may also be of value to tES researchers in fields other than attention.

The large variability in stimulation parameters is one important factor that may explain the lack of consistent resultswe rarely came across two studies that used the same protocol (Tables 1, 2, 3, 4, and 5). Electrode montage and stimulation intensity, duration, timing, and polarity alone offer a daunting number of degrees of freedom, and all of these parameters can greatly affect the outcome of stimulation. For instance, varying stimulation duration (Monte-Silva et al. 2013) or current intensity (Batsikadze et al. 2013) may completely flip the effect of tDCS between excitation and inhibition. Even the order of sham and real tES sessions could potentially affect the outcome: tES may interact with practice-related improvements in task performance, for example, such that tES effects are less pronounced in later sessions. This remains a factor even if session order is counterbalanced between subjects.

Mostly, this review highlights a dire need for studies that more systematically explore the parameter space and for a mechanistic understanding of the neurophysiological effects of tES. To determine promising parameter combinations, direct replications are essential. Preregistration may also facilitate progress in the field, as a recent meta-analysis of tDCS and working memory found some evidence for selective reporting of positive results (Mancuso et al. 2016).

Future studies should also make an effort to increase power: some studies had particularly low sample sizes (fewer than 10 participants per group) or trial counts (fewer than $10 \mathrm{~min}$ of task performance), which do little to mitigate within- and between-subject variability. Indeed, several studies underlined that individual differences may shape the outcome of stimulation. Many factors could play a role here, ranging from differences in head and brain anatomy to gender and genetics (see Li et al. (2015b) and Krause and Cohen Kadosh (2014) for reviews). Differences in baseline brain state and cortical excitation/inhibition balance seem especially relevant (Krause et al. 2013), as they could explain why in some studies the effects of stimulation were contingent on baseline task performance (e.g., Learmonth et al. 2015; Benwell et al. 2015; London and Slagter 2015).

Recent studies have shown that even the influence of stimulation on motor-evoked potentials - the primary proof of tDCS efficacy in humans - is subject to high inter- (López-Alonso et al. 2014; Strube et al. 2016; Wiethoff et al. 2014) and intraindividual variability (Dyke et al. 2016; Horvath et al. 2015b; López-Alonso et al. 2015). The ultimate solution may be to tailor stimulation dosage and placement of electrodes to individual brains, but this requires sophisticated computational modeling efforts that are only just getting under way (Berker et al. 2013; Bikson et al. 2012).

Understanding the factors that drive tES responsiveness is absolutely crucial to the aim of cognitive enhancement. One cannot meaningfully speak of enhancement when a substantial portion of individuals shows no response or even a detriment. Potential costs to cognitive enhancement are often overlooked, but are a real possibility: enhancement of one cognitive function could be paired with a decline in another function (Brem et al. 2014a; Iuculano and Cohen Kadosh 2013; Sarkar et al. 2014), as the brain networks underlying cognitive functions do not operate in isolation (Wokke et al. 2015).

In the diseased brain, this principle may be exploited to return network function to the normal state. For instance, in hemispatial neglect, tDCS studies may restore the balance 
between the overactive, non-lesioned hemisphere and the overinhibited, lesioned hemisphere. However, in the healthy brain, boosting one network function with tES may incur a cost to another network function. For example, while cathodal parietal tDCS enhanced attention to ipsilateral stimuli, it worsened performance for contralateral and bilateral stimuli (Filmer et al. 2015; Sparing et al. 2009). Similarly, an improved ability to focus attention in a top-down manner (e.g., on the road when driving) may hamper bottom-up attention to unexpected, albeit potentially relevant stimuli (e.g., a child next to the road).

To evaluate such costs, including control tasks that probe other cognitive abilities is essential (Parkin et al. 2015; Wokke et al. 2015). This is particularly important for multiple session tES studies, where both enhancements and costs may be larger and potentially longer lasting. Future research in this direction is imperative, as virtually nothing is known about the longterm effects of repeated tES on attention processes in the healthy brain, nor about its potential adverse effects.

Another important avenue for future research is to combine tES with neuroimaging techniques (Bergmann et al. 2016). Concurrent applications of tES with fMRI or M/EEG measurements are technically challenging but allow for more insight into the neurophysiology of tES and the relationship between baseline activity and tES effects. Moreover, neuroimaging may greatly inform the choice of stimulation parameters. Clark et al. (2012) determined the stimulation site based on a prior fMRI study, which may have contributed to the large and consistent effects they found. Similarly, prior M/ EEG studies may aid in picking the most optimal stimulation frequency (van Driel et al. 2015). Future studies combining tACS with M/EEG are also necessary to determine if tACSby synchronizing endogenous neural oscillations - may be particularly effective for enhancing attention.

Is transcranial electrical stimulation an effective tool to enhance attention? At present, it is too early to say. Although the initial findings are encouraging, they require replication and further study. However, the interest from society at large in tES is considerable and has grossly outpaced the state of the field. Informal surveys suggest that enhancement is the most common incentive for the growing use of tES at home, and that attention tops the list of enhancement purposes (Jwa 2015). A recent open letter signed by many leading tES experts commands utmost caution and highlights a long list of unknowns that preclude practical applications of tES for enhancement (Wurzman et al. 2016). We can only underscore that statement on the basis of this review and hope that our overview of current studies and recommendations for future research will help to determine the efficacy of tES for enhancing attention.

Acknowledgements We thank Marlies Vissers and three anonymous reviewers for providing useful feedback on the manuscript. This work was supported by a VIDI and a Research Talent grant from the Netherlands Organization for Scientific Research (NWO) to HAS and a Marie Curie European Fellowship grant to MRvS.

Open Access This article is distributed under the terms of the Creative Commons Attribution 4.0 International License (http:// creativecommons.org/licenses/by/4.0/), which permits unrestricted use, distribution, and reproduction in any medium, provided you give appropriate credit to the original author(s) and the source, provide a link to the Creative Commons license, and indicate if changes were made.

\section{References}

Antal, A., \& Herrmann, C. S. (2016). Transcranial alternating current and random noise stimulation: possible mechanisms. Neural Plasticity, 2016, 1-12. doi:10.1155/2016/3616807.

Axelrod, V., Rees, G., Lavidor, M., \& Bar, M. (2015). Increasing propensity to mind-wander with transcranial direct current stimulation. Proceedings of the National Academy of Sciences of the United States of America, 112(11), 3314-3319. doi:10.1073/pnas. 1421435112.

Ball, K. L., Lane, A. R., Smith, D. T., \& Ellison, A. (2013). Sitedependent effects of tDCS uncover dissociations in the communication network underlying the processing of visual search. Brain stimulation, 6, 959-965. doi:10.1016/j.brs.2013.06.001.

Bang, D.-H., \& Bong, S.-Y. (2015). Effect of combination of transcranial direct current stimulation and feedback training on visuospatial neglect in patients with subacute stroke: a pilot randomized controlled trial. Journal of Physical Therapy Science, 27(9), 2759-2761. doi: 10.1589/jpts.27.2759.

Bardi, L., Kanai, R., Mapelli, D., \& Walsh, V. (2013). Direct current stimulation (tDCS) reveals parietal asymmetry in local/global and salience-based selection. Cortex, 49(3), 850-860. doi:10.1016/j. cortex.2012.04.016.

Batsikadze, G., Moliadze, V., Paulus, W., Kuo, M.-F., \& Nitsche, M. A. (2013). Partially non-linear stimulation intensity-dependent effects of direct current stimulation on motor cortex excitability in humans. The Journal of Physiology, 591(Pt 7), 1987-2000. doi:10.1113/ jphysiol.2012.249730.

Benwell, C. S. Y., Learmonth, G., Miniussi, C., Harvey, M., \& Thut, G. (2015). Non-linear effects of transcranial direct current stimulation as a function of individual baseline performance: evidence from biparietal tDCS influence on lateralized attention bias. Cortex, 69 , 152-165. doi:10.1016/j.cortex.2015.05.007.

Bergmann, T. O., Karabanov, A., Hartwigsen, G., Thielscher, A., \& Siebner, H. R. (2016). Combining non-invasive transcranial brain stimulation with neuroimaging and electrophysiology: current approaches and future perspectives. NeuroImage, 140, 4-19. doi:10. 1016/j.neuroimage.2016.02.012.

Bestmann, S., de Berker, A. O., \& Bonaiuto, J. (2015). Understanding the behavioural consequences of noninvasive brain stimulation. Trends in Cognitive Sciences, 19(1), 13-20. doi:10.1016/j.tics.2014.10.003.

Bikson, M., Rahman, A., \& Datta, A. (2012). Computational models of transcranial direct current stimulation. Clinical EEG and Neuroscience, 43(3), 176-183. doi:10.1177/1550059412445138.

Bindman, L. J., Lippold, O. C. J., \& Redfearn, J. W. T. (1964). The action of brief polarizing currents on the cerebral cortex of the rat (1) during current flow and (2) in the production of long-lasting after effects. Journal of Physiology, 172, 369-382. doi:10.1113/jphysiol.1964. sp007425.

Blumberg, E. J., Peterson, M. S., \& Parasuraman, R. (2015). Enhancing multiple object tracking performance with noninvasive brain stimulation: a causal role for the anterior intraparietal sulcus. Frontiers in 
Systems Neuroscience, 9(February), 3. doi:10.3389/fnsys.2015. 00003.

Bolognini, N., Fregni, F., Casati, C., Olgiati, E., \& Vallar, G. (2010). Brain polarization of parietal cortex augments training-induced improvement of visual exploratory and attentional skills. Brain Research, 1349, 76-89. doi:10.1016/j.brainres.2010.06.053.

Bolognini, N., Olgiati, E., Rossetti, A., \& Maravita, A. (2010). Enhancing multisensory spatial orienting by brain polarization of the parietal cortex. The European Journal of Neuroscience, 31(10), 1800-1806. doi:10.1111/j.1460-9568.2010.07211.x.

Bowers, D., \& Heilman, K. M. (1980). Pseudoneglect: effects of hemispace on a tactile line bisection task. Neuropsychologia, 18(45), 491-498. doi:10.1016/0028-3932(80)90151-7.

Brem, A.-K., Fried, P. J., Horvath, J. C., Robertson, E. M., \& PascualLeone, A. (2014). Is neuroenhancement by noninvasive brain stimulation a net zero-sum proposition? NeuroImage, 85, 1058-1068. doi:10.1016/j.neuroimage.2013.07.038.

Brem, A.-K., Unterburger, E., Speight, I., \& Jäncke, L. (2014). Treatment of visuospatial neglect with biparietal tDCS and cognitive training: a single-case study. Frontiers in Systems Neuroscience, 8(September), 180. doi:10.3389/fnsys.2014.00180.

Brignani, D., Ruzzoli, M., Mauri, P., \& Miniussi, C. (2013). Is transcranial alternating current stimulation effective in modulating brain oscillations? PLOS ONE, 8(2), e56589. doi:10.1371/journal.pone. 0056589.

Buschman, T. J., \& Miller, E. K. (2007). Top-down versus bottom-up control of attention in the prefrontal and posterior parietal cortices. Science, 315(5820), 1860-1862. doi:10.1126/science.1138071.

Buzsáki, G., \& Draguhn, A. (2004). Neuronal oscillations in cortical networks. Science, 304(5679), 1926-1929. doi:10.1126/science. 1099745.

Callan, D. E., Falcone, B., Wada, A., \& Parasuraman, R. (2016). Simultaneous tDCS-fMRI identifies resting state networks correlated with visual search enhancement. Frontiers in Human Neuroscience, 10(March), 72. doi:10.3389/fnhum.2016.00072.

Chica, A. B., Martín-Arévalo, E., Botta, F., \& Lupiáñez, J. (2014). The spatial orienting paradigm: how to design and interpret spatial attention experiments. Neuroscience and Biobehavioral Reviews, 40, 35 51. doi:10.1016/j.neubiorev.2014.01.002.

Clark, V. P., Coffman, B. A., Trumbo, M. C., \& Gasparovic, C. (2011). Transcranial direct current stimulation (tDCS) produces localized and specific alterations in neurochemistry: a $1 \mathrm{H}$ magnetic resonance spectroscopy study. Neuroscience Letters, 500(1), 67-71. doi:10. 1016/j.neulet.2011.05.244.

Clark, V. P., Coffman, B. A., Mayer, A. R., Weisend, M. P., Lane, T. D. R., Calhoun, V. D., et al. (2012). TDCS guided using fMRI significantly accelerates learning to identify concealed objects. NeuroImage, 59(1), 117-128. doi:10.1016/j.neuroimage.2010.11.036.

Coffman, B. A., Trumbo, M. C., \& Clark, V. P. (2012). Enhancement of object detection with transcranial direct current stimulation is associated with increased attention. BMC Neuroscience, 13, 108. doi:10. 1186/1471-2202-13-108.

Coffman, B. A., Clark, V. P., \& Parasuraman, R. (2014). Battery powered thought: enhancement of attention, learning, and memory in healthy adults using transcranial direct current stimulation. NeuroImage, 85(Pt 3), 895-908. doi:10.1016/j.neuroimage.2013.07.083.

Cohen Kadosh, R. (Ed.). (2014). The stimulated brain: cognitive enhancement using non-invasive brain stimulation. London: Academic.

Corbetta, M., \& Shulman, G. L. (2002). Control of goal-directed and stimulus-driven attention in the brain. Nature Reviews Neuroscience, 3(3), 201-215. doi:10.1038/nrn755.

Corbetta, M., Kincade, M. J., Lewis, C., Snyder, A. Z., \& Sapir, A. (2005). Neural basis and recovery of spatial attention deficits in spatial neglect. Nature Neuroscience, 8(11), 1603-1610. doi:10. 1038/nn1574.
Cosman, J. D., Atreya, P. V., \& Woodman, G. F. (2015). Transient reduction of visual distraction following electrical stimulation of the prefrontal cortex. Cognition, 145, 73-76. doi:10.1016/j.cognition. 2015.08.010.

de Berker, A. O., Bikson, M., \& Bestmann, S. (2013). Predicting the behavioral impact of transcranial direct current stimulation: issues and limitations. Frontiers in Human Neuroscience, 7(October), 613. doi:10.3389/fnhum.2013.00613.

de Tommaso, M., Invitto, S., Ricci, K., Lucchese, V., Delussi, M., Quattromini, P., et al. (2014). Effects of anodal TDCS stimulation of left parietal cortex on visual spatial attention tasks in men and women across menstrual cycle. Neuroscience Letters, 574, 21-25. doi:10.1016/j.neulet.2014.05.014.

Dyke, K., Kim, S., Jackson, G. M., \& Jackson, S. R. (2016). Intra-subject consistency and reliability of response following $2 \mathrm{~mA}$ transcranial direct current stimulation. Brain Stimulation, 1-7. doi:10.1016/j.brs. 2016.06.052.

Ellison, A., Ball, K. L., Moseley, P., Dowsett, J., Smith, D. T., Weis, S., et al. (2014). Functional interaction between right parietal and bilateral frontal cortices during visual search tasks revealed using functional magnetic imaging and transcranial direct current stimulation. PLOS ONE, 9(4), e93767. doi:10.1371/journal.pone.0093767.

Falcone, B., Coffman, B. A., Clark, V. P., \& Parasuraman, R. (2012). Transcranial direct current stimulation augments perceptual sensitivity and 24-hour retention in a complex threat detection task. PLOS ONE, 7(4), e34993. doi:10.1371/journal.pone.0034993.

Fan, J., McCandliss, B. D., Sommer, T., Raz, A., \& Posner, M. I. (2002). Testing the efficiency and independence of attentional networks. Journal of Cognitive Neuroscience, 14(3), 340-347. doi:10.1162/ 089892902317361886.

Filmer, H. L., Dux, P. E., \& Mattingley, J. B. (2015). Dissociable effects of anodal and cathodal tDCS reveal distinct functional roles for right parietal cortex in the detection of single and competing stimuli. Neuropsychologia, 74, 120-126. doi:10.1016/j.neuropsychologia. 2015.01.038.

Giglia, G., Mattaliano, P., Puma, A., Rizzo, S., Fierro, B., \& Brighina, F. (2011). Neglect-like effects induced by tDCS modulation of posterior parietal cortices in healthy subjects. Brain Stimulation, 4(4), 294-299. doi:10.1016/j.brs.2011.01.003.

Green, C. S., \& Bavelier, D. (2012). Learning, attentional control, and action video games. Current Biology, 22(6), R197-R206. doi:10. 1016/j.cub.2012.02.012.

Heilman, K. M., Watson, R., \& Valenstein, E. (2012). Neglect and related disorders. In K. Heilman \& E. Valenstein (Eds.), Clinical neurophysiology (5th ed., pp. 296-348). New York: Oxford University Press.

Herrmann, C. S., Rach, S., Neuling, T., \& Strüber, D. (2013). Transcranial alternating current stimulation: a review of the underlying mechanisms and modulation of cognitive processes. Frontiers in Human Neuroscience, 7(June), 279. doi:10.3389/fnhum.2013.00279.

Hone-Blanchet, A., Edden, R. A., \& Fecteau, S. (2016). Online effects of transcranial direct current stimulation in real time on human prefrontal and striatal metabolites. Biological Psychiatry, 80(6), 432-438. doi:10.1016/j.biopsych.2015.11.008.

Hopfinger, J. B., Parsons, J., \& Fröhlich, F. (2016). Differential effects of $10-\mathrm{Hz}$ and $40-\mathrm{Hz}$ transcranial alternating current stimulation (tACS) on endogenous versus exogenous attention. Cognitive Neuroscience, 8928(June), 1-10. doi:10.1080/17588928.2016. 1194261.

Horvath, J. C., Forte, J. D., \& Carter, O. (2015). Quantitative review finds no evidence of cognitive effects in healthy populations from singlesession transcranial direct current stimulation (tDCS). Brain Stimulation, 8(3), 535-550. doi:10.1016/j.brs.2015.01.400.

Horvath, J. C., Vogrin, S. J., Carter, O., Cook, M. J., \& Forte, J. D. (2015). Effects of transcranial direct current stimulation on motor evoked potential amplitude are neither reliable nor significant within 
individuals over 9 separate testing sessions. Brain Stimulation, 8(2), 318. doi:10.1016/j.brs.2015.01.033.

Hsu, W.-Y., Zanto, T. P., Anguera, J. A., Lin, Y.-Y., \& Gazzaley, A. (2015). Delayed enhancement of multitasking performance: effects of anodal transcranial direct current stimulation on the prefrontal cortex. Cortex, 69, 175-185. doi:10.1016/j.cortex.2015.05.014.

Iuculano, T., \& Cohen Kadosh, R. (2013). The mental cost of cognitive enhancement. Journal of Neuroscience, 33(10), 4482-4486. doi:10. 1523/JNEUROSCI.4927-12.2013.

Jacobson, L., Koslowsky, M., \& Lavidor, M. (2012). tDCS polarity effects in motor and cognitive domains: a meta-analytical review. Experimental Brain Research, 216(1), 1-10. doi:10.1007/s00221011-2891-9.

Jewell, G., \& McCourt, M. E. (2000). Pseudoneglect: a review and metaanalysis of performance factors in line bisection tasks. Neuropsychologia, 38(1), 93-110. doi:10.1016/S0028-3932(99) 00045-7.

Jones, K. T., \& Berryhill, M. E. (2012). Parietal contributions to visual working memory depend on task difficulty. Frontiers in Psychiatry, 3, 1-11. doi:10.3389/fpsyt.2012.00081.

Jwa, A. (2015). Early adopters of the magical thinking cap: a study on doit-yourself (DIY) transcranial direct current stimulation (tDCS) user community. Journal of Law and the Biosciences. 1-44. doi:10.1093/ $\mathrm{jlb} / \mathrm{lsv} 017$.

Kajimura, S., \& Nomura, M. (2015). Decreasing propensity to mindwander with transcranial direct current stimulation. Neuropsychologia, 75, 533-537. doi:10.1016/j.neuropsychologia. 2015.07.013.

Kajimura, S., Kochiyama, T., Nakai, R., Abe, N., \& Nomura, M. (2016). Causal relationship between effective connectivity within the default mode network and mind-wandering regulation and facilitation. NeuroImage, 133, 21-30. doi:10.1016/j.neuroimage.2016.03.009.

Ko, M.-H., Han, S.-H., Park, S.-H., Seo, J.-H., \& Kim, Y.-H. (2008). Improvement of visual scanning after DC brain polarization of parietal cortex in stroke patients with spatial neglect. Neuroscience Letters, 448(2), 171-174. doi:10.1016/j.neulet.2008.10.050.

Koelega, H. S. (1993). Stimulant drugs and vigilance performance: a review. Psychopharmacology, 111(1), 1-16. doi:10.1007/ BF02257400.

Krause, B., \& Cohen Kadosh, R. (2014). Not all brains are created equal: the relevance of individual differences in responsiveness to transcranial electrical stimulation. Frontiers in systems neuroscience, 8(February), 25. doi:10.3389/fnsys.2014.00025.

Krause, B., Márquez-Ruiz, J., \& Kadosh, R. C. (2013). The effect of transcranial direct current stimulation: a role for cortical excitation/ inhibition balance? Frontiers in Human Neuroscience, 7(September), 602. doi:10.3389/fnhum.2013.00602.

Laczó, B., Antal, A., Niebergall, R., Treue, S., \& Paulus, W. (2012). Transcranial alternating stimulation in a high gamma frequency range applied over V1 improves contrast perception but does not modulate spatial attention. Brain stimulation, 5(4), 484-491. doi:10. 1016/j.brs.2011.08.008.

Làdavas, E., Giulietti, S., Avenanti, A., Bertini, C., Lorenzini, E., Quinquinio, C., et al. (2015). A-tDCS on the ipsilesional parietal cortex boosts the effects of prism adaptation treatment in neglect. Restorative Neurology and Neuroscience, 33(5), 647-662. doi:10. 3233/RNN-140464.

Learmonth, G., Thut, G., Benwell, C. S. Y., \& Harvey, M. (2015). The implications of state-dependent tDCS effects in aging: behavioural response is determined by baseline performance. Neuropsychologia, 74, 108-119. doi:10.1016/j.neuropsychologia.2015.01.037.

Li, L. M., Leech, R., Scott, G., Malhotra, P., Seemungal, B., \& Sharp, D. J. (2015). The effect of oppositional parietal transcranial direct current stimulation on lateralized brain functions. European Journal of Neuroscience, 42(11), 2904-2914. doi:10.1111/ejn.13086.
Li, L. M., Uehara, K., \& Hanakawa, T. (2015). The contribution of interindividual factors to variability of response in transcranial direct current stimulation studies. Frontiers in Cellular Neuroscience, 9(May), 181. doi:10.3389/fncel.2015.00181.

Loftus, A. M., \& Nicholls, M. E. R. (2012). Testing the activationorientation account of spatial attentional asymmetries using transcranial direct current stimulation. Neuropsychologia, 50(11), 2573 2576. doi:10.1016/j.neuropsychologia.2012.07.003.

London, R. E., \& Slagter, H. A. (2015). Effects of transcranial direct current stimulation over left dorsolateral $\mathrm{pFC}$ on the attentional blink depend on individual baseline performance. Journal of Cognitive Neuroscience, 27(12), 2382-2393. doi:10.1162/jocn_a_00867.

López-Alonso, V., Cheeran, B., Río-Rodríguez, D., \& Fernández-DelOlmo, M. (2014). Inter-individual variability in response to noninvasive brain stimulation paradigms. Brain Stimulation, 7(3), 372-380. doi:10.1016/j.brs.2014.02.004.

López-Alonso, V., Fernández-del-Olmo, M., Costantini, A., GonzalezHenriquez, J. J., \& Cheeran, B. (2015). Intra-individual variability in the response to anodal transcranial direct current stimulation. Clinical Neurophysiology, 126(12), 2342-2347. doi:10.1016/j. clinph.2015.03.022.

Lutz, A., Slagter, H. A., Dunne, J. D., \& Davidson, R. J. (2008). Attention regulation and monitoring in meditation. Trends in Cognitive Sciences, 12(4), 163-169. doi:10.1016/j.tics.2008.01.005.

Mackworth, N. (1948). The breakdown of vigilance durning prolonged visual search. Quarterly Journal of Experimental Psychology, 1, 621.

Mancuso, L. E., Ilieva, I. P., Hamilton, R. H., \& Farah, M. J. (2016). Does transcranial direct current stimulation improve healthy working memory?: a meta-analytic review. Journal of Cognitive Neuroscience, 28(8), 1063-1089. doi:10.1162/jocn a 00956.

Mauri, P., Miniussi, C., Balconi, M., \& Brignani, D. (2015). Bursts of transcranial electrical stimulation increase arousal in a continuous performance test. Neuropsychologia, 74, 127-136. doi:10.1016/j. neuropsychologia.2015.03.006.

McIntire, L. K., McKinley, R. A., Goodyear, C., \& Nelson, J. M. (2014). A comparison of the effects of transcranial direct current stimulation and caffeine on vigilance and cognitive performance during extended wakefulness. Brain Stimulation, 7(4), 499-507. doi:10.1016/j. brs.2014.04.008.

Medeiros, L. F., de Souza, I. C. C., Vidor, L. P., de Souza, A., Deitos, A., Volz, M. S., et al. (2012). Neurobiological effects of transcranial direct current stimulation: a review. Frontiers in Psychiatry, 3(December), 110. doi:10.3389/fpsyt.2012.00110.

Medina, J., Beauvais, J., Datta, A., Bikson, M., Coslett, H. B., \& Hamilton, R. H. (2013). Transcranial direct current stimulation accelerates allocentric target detection. Brain Stimulation, 6(3), 433439. doi:10.1016/j.brs.2012.05.008.

Miller, J., Berger, B., \& Sauseng, P. (2015). Anodal transcranial direct current stimulation (tDCS) increases frontal-midline theta activity in the human EEG: a preliminary investigation of non-ianvasive stimulation. Neuroscience Letters, 588, 114-119. doi:10.1016/j.neulet. 2015.01.014.

Monte-Silva, K., Kuo, M.-F., Hessenthaler, S., Fresnoza, S., Liebetanz, D., Paulus, W., et al. (2013). Induction of late LTP-like plasticity in the human motor cortex by repeated non-invasive brain stimulation. Brain Stimulation, 6(3), 424-432. doi:10.1016/j.brs.2012.04.011.

Moos, K., Vossel, S., Weidner, R., Sparing, R., \& Fink, G. R. (2012). Modulation of top-down control of visual attention by cathodal tDCS over right IPS. Journal of Neuroscience, 32(46), 1636016368. doi:10.1523/JNEUROSCI.6233-11.2012.

Müller, N. G., Vellage, A. K., Heinze, H. J., \& Zaehle, T. (2015). Entrainment of human alpha oscillations selectively enhances visual conjunction search. PLOS ONE, 10(11), e0143533. doi:10.1371/ journal.pone.0143533. 
Nelson, J. T., McKinley, R. A., Golob, E. J., Warm, J. S., \& Parasuraman, R. (2014). Enhancing vigilance in operators with prefrontal cortex transcranial direct current stimulation (tDCS). NeuroImage, 85, 909-917. doi:10.1016/j.neuroimage.2012.11.061.

Nieratschker, V., Kiefer, C., Giel, K., Kruger, R., \& Plewnia, C. (2015). The COMT Val/Met polymorphism modulates effects of tDCS on response inhibition. Brain Stimulation, 8(2), 283-288. doi:10.1016/ j.brs.2014.11.009.

Nitsche, M. A., \& Paulus, W. (2000). Excitability changes induced in the human motor cortex by weak transcranial direct current stimulation. The Journal of Physiology, 527 Pt 3(2000), 633-639. doi:10.1111/j. 1469-7793.2000.t01-1-00633.x.

Nitsche, M. A., \& Paulus, W. (2001). Sustained excitability elevations induced by transcranial DC motor cortex stimulation in humans. Neurology, 57(10), 1899-1901. doi:10.1212/WNL.57.10.1899.

Nitsche, M. A., Cohen, L. G., Wassermann, E. M., Priori, A., Lang, N., Antal, A., et al. (2008). Transcranial direct current stimulation: state of the art 2008. Brain Stimulation, 1(3), 206-223. doi:10.1016/j.brs. 2008.06.004.

Opitz, A., Paulus, W., Will, S., Antunes, A., \& Thielscher, A. (2015). Determinants of the electric field during transcranial direct current stimulation. Neurolmage, 109, 140-150. doi:10.1016/j.neuroimage. 2015.01.033.

Parasuraman, R. (1979). Memory load and event rate control sensitivity decrements in sustained attention. Science, 205(4409), 924-927. doi:10.1126/science.472714.

Parkin, B. L., Ekhtiari, H., \& Walsh, V. F. (2015). Non-invasive human brain stimulation in cognitive neuroscience: a primer. Neuron, 87(5), 932-945. doi:10.1016/j.neuron.2015.07.032.

Picazio, S., Granata, C., Caltagirone, C., Petrosini, L., \& Oliveri, M. (2015). Shaping pseudoneglect with transcranial cerebellar direct current stimulation and music listening. Frontiers in Human Neuroscience, 9(March), 1-9. doi:10.3389/fnhum.2015.00158.

Plewnia, C., Zwissler, B., Längst, I., Maurer, B., Giel, K., \& Krüger, R. (2013). Effects of transcranial direct current stimulation (tDCS) on executive functions: influence of COMT Val/Met polymorphism. Cortex, 49(7), 1801-1807. doi:10.1016/j.cortex.2012.11.002.

Posner, M. I. (1980). Orienting of attention. The Quarterly Journal of Experimental Psychology, 32(1), 3-25.

Radman, T., Ramos, R. L., Brumberg, J. C., \& Bikson, M. (2009). Role of cortical cell type and morphology in subthreshold and suprathreshold uniform electric field stimulation in vitro. Brain Stimulation, 2, 215-228. doi:10.1016/j.brs.2009.03.007.

Reinhart, R. M. G., \& Woodman, G. F. (2015). Enhancing long-term memory with stimulation tunes visual attention in one trial. Proceedings of the National Academy of Sciences of the United States of America, 112(2), 625-630. doi:10.1073/pnas.1417259112.

Reynolds, J. H., \& Chelazzi, L. (2004). Attentional modulation of visual processing. Annual Review of Neuroscience, 27, 611-647. doi:10. 1146/annurev.neuro.26.041002.131039.

Roe, J. M., Nesheim, M., Mathiesen, N. C., Moberget, T., Alnæs, D., \& Sneve, M. H. (2016). The effects of tDCS upon sustained visual attention are dependent on cognitive load. Neuropsychologia, 80, 1-8. doi:10.1016/j.neuropsychologia.2015.11.005.

Roy, L. B., Sparing, R., Fink, G. R., \& Hesse, M. D. (2015). Modulation of attention functions by anodal tDCS on right PPC. Neuropsychologia, 74, 96-107. doi:10.1016/j.neuropsychologia. 2015.02.028.

Sarkar, A., Dowker, A., \& Cohen Kadosh, R. (2014). Cognitive enhancement or cognitive cost: trait-specific outcomes of brain stimulation in the case of mathematics anxiety. Journal of Neuroscience, 34(50), 16605-16610. doi:10.1523/JNEUROSCI.3129-14.2014.

Siegel, M., \& Donner, T. (2010). Linking band-limited cortical activity to fMRI and behavior. In Simultaneous eeg and fMRI. recording, analysis and application (1-23). New York: Oxford University Press. doi:10.1093/acprof:oso/9780195372731.003.0017.
Smit, M., Schutter, D. J. L. G., Nijboer, T. C. W., Visser-Meily, J. M. A., Kappelle, L. J., Kant, N., et al. (2015). Transcranial direct current stimulation to the parietal cortex in hemispatial neglect: a feasibility study. Neuropsychologia, 74, 152-161. doi:10.1016/j. neuropsychologia.2015.04.014.

Snowball, A., Tachtsidis, I., Popescu, T., Thompson, J., Delazer, M., Zamarian, L., et al. (2013). Long-term enhancement of brain function and cognition using cognitive training and brain stimulation. Current Biology, 23(11), 987-992. doi:10.1016/j.cub.2013.04.045.

Sparing, R., Thimm, M., Hesse, M. D., Küst, J., Karbe, H., \& Fink, G. R. (2009). Bidirectional alterations of interhemispheric parietal balance by non-invasive cortical stimulation. Brain, 132(Pt 11), 3011-3020. doi:10.1093/brain/awp154.

Stagg, C. J., \& Nitsche, M. A. (2011). Physiological basis of transcranial direct current stimulation. The Neuroscientist, 17(1), 37-53. doi:10. 1177/1073858410386614.

Stagg, C. J., Best, J. G., Stephenson, M. C., O'Shea, J., Wylezinska, M., Kincses, Z. T., et al. (2009). Polarity-sensitive modulation of cortical neurotransmitters by transcranial stimulation. Journal of Neuroscience, 29(16), 5202-5206. doi:10.1523/JNEUROSCI. 4432-08.2009.

Stone, D. B., \& Tesche, C. D. (2009). Transcranial direct current stimulation modulates shifts in global/local attention. Neuroreport, 20(12), 1115-1119. doi:10.1097/WNR.0b013e32832e9aa2.

Strube, W., Bunse, T., Nitsche, M. A., Nikolaeva, A., Palm, U., Padberg, F., et al. (2016). Bidirectional variability in motor cortex excitability modulation following $1 \mathrm{~mA}$ transcranial direct current stimulation in healthy participants. Physiological Reports, 4(15), e12884. doi:10. 14814/phy2.12884.

Sunwoo, H., Kim, Y. H., Chang, W. H., Noh, S., Kim, E. J., \& Ko, M.-H. (2013). Effects of dual transcranial direct current stimulation on post-stroke unilateral visuospatial neglect. Neuroscience Letters, 554, 94-98. doi:10.1016/j.neulet.2013.08.064.

Terney, D., Chaieb, L., Moliadze, V., Antal, A., \& Paulus, W. (2008). Increasing human brain excitability by transcranial high-frequency random noise stimulation. Journal of Neuroscience, 28(52), 1414714155. doi:10.1523/JNEUROSCI.4248-08.2008.

Terzuolo, C., \& Bullock, T. (1956). Measurement of imposed voltage gradient adequate to modulate neuronal firing. Proceedings of the National Academy of Sciences of the United States of America, 42, 687-694.

Turgut, N., Miranda, M., Kastrup, A., Eling, P., \& Hildebrandt, H. (2016). tDCS combined with optokinetic drift reduces egocentric neglect in severely impaired post-acute patients. Neuropsychological Rehabilitation, 2011. doi:10.1080/09602011.2016.1202120.

Vallar, G., \& Perani, D. (1986). The anatomy of unilateral neglect after right-hemisphere stroke lesions. A clinical/CT-scan correlation study in man. Neuropsychologia, 24(5), 609-622. doi:10.1016/ 0028-3932(86)90001-1.

van Driel, J., Sligte, I. G., Linders, J., Elport, D., \& Cohen, M. X. (2015). Frequency band-specific electrical brain stimulation modulates cognitive control processes. PLOS ONE, 10(9), e0138984. doi:10.1371/ journal.pone.0138984.

Weiss, M., \& Lavidor, M. (2012). When less is more: evidence for a facilitative cathodal tDCS effect in attentional abilities. Journal of Cognitive Neuroscience, 24(9), 1826-1833. doi:10.1162/jocn_a 00248.

Wiethoff, S., Hamada, M., \& Rothwell, J. C. (2014). Variability in response to transcranial direct current stimulation of the motor cortex. Brain stimulation, 7(3), 468-475. doi:10.1016/j.brs.2014.02.003.

Wokke, M. E., Talsma, L. J., \& Vissers, M. E. (2015). Biasing neural network dynamics using non-invasive brain stimulation. Frontiers in Systems Neuroscience, 8(January), 1-5. doi:10.3389/fnsys.2014. 00246. 
Wolfe, J. M. (1998). Visual search. In H. Pashler (Ed.), Attention. London: University College London Press. doi:10.1016/j.tics. 2010.12.001.

Womelsdorf, T., \& Fries, P. (2007). The role of neuronal synchronization in selective attention. Current Opinion in Neurobiology, 17(2), 154 160. doi:10.1016/j.conb.2007.02.002.

Wright, J. M., \& Krekelberg, B. (2014). Transcranial direct current stimulation over posterior parietal cortex modulates visuospatial localization. Journal of Vision, 14, 1-15. doi:10.1167/14.9.5.doi.
Wurzman, R., Hamilton, R., Pascual-Leone, A., \& Fox, M. (2016). An open letter concerning do-it-yourself (DIY) users of transcranial direct current stimulation (tDCS). Annals of Neurology, 1-4. doi: 10.1002/ana.24689.

Yi, Y. G., Chun, M. H., Do, K. H., Sung, E. J., Kwon, Y. G., \& Kim, D. Y. (2016). The effect of transcranial direct current stimulation on neglect syndrome in stroke patients. Annals of Rehabilitation Medicine, 40(2), 223-229. doi:10.5535/arm.2016.40.2.223. 